\title{
Veranstaltungsübersicht 2021
}

\section{Kongresse 2021}

Seminarwoche:

70. Seminarwoche Innere Medizin kompakt

Pörtschach am Wörtersee, Österreich, 29.8.2021-3.9.2021,

vorauss. 40 DFP Punkte

Leitung:

Prof. Dr. med. Andreas Tromm, Hattingen

Kongressort: Prof. Dr. med. Bernhard Wörmann, Berlin

Congress Center Wörthersee

Hauptstr. 203, 9210 Pörtschach a. Wörthersee

Österreich

Teilnahmegebühr: $\quad$ Seminarwoche $620 €$ Nicht-Mitglied, $341 €$ BDI-Mitglied

\section{Kurse 2021}

Intensivkurs:

Pneumologie - Update 2021

Bonn, 11.-12.6.2021, CME vorauss. 16

Kursleitung: $\quad$ PD Dr. med. Selcuk Tasci, Siegburg

Kursort: $\quad$ Universitätsclub Bonn e.V.

Konviktstr. 9, 53113 Bonn

Teilnahmegebühr: Präsenz: $660 €$ Nicht-Mitglied, $363 €$ BDI Mitglied

Livestream: $560 €$ Nicht-Mitglied, $308 €$ BDI-Mitglied

BDI Online:

Volkskrankheit der Inneren Medizin

Online, 16.6.2021, CME vorauss. 3

Leitung: Prof. Dr. med. Ahmed Madisch, Hannover

Teilnahme: kostenfrei

Intensivkurs:

Allgemeine Innere Medizin - Was ist neu 2021?

Berlin, 18.-20.6.2021, CME vorauss. 16

Kursleitung: $\quad$ Prof. Dr. med. Bernhard Wörmann, Berlin

Kursort: $\quad$ Karl Storz SE \& Co. KG

Besucher- und Schulungszentrum Berlin

Scharnhorststr. 3, 10115 Berlin (Mitte)

Teilnahmegebühr: $\quad$ Präsenz: $660 €$ Nicht-Mitglied, $363 € B D I$ Mitglied

Livestream: $560 €$ Nicht-Mitglied, $308 €$ BDI-Mitglied

Intensivkurs:

Nephrologie/Diabetologie - Update 2021

Weimar, 25.-26.6.2021, CME vorauss. 16

Kursleitung: $\quad$ Prof. Dr. med. Reinhard Fünfstück, Weimar

PD Dr. med. Kornelia Konz, Wiesbaden

Kursort: $\quad$ Dorint Am Goethepark Weimar

Beethovenplatz 1/2, 99423 Weimar

Teilnahmegebühr: Präsenz: $660 €$ Nicht-Mitglied, $363 €$ BDI Mitglied

Livestream: $560 €$ Nicht-Mitglied, $308 €$ BDI-Mitglied

Praktischer Kurs:

Farbdoppler-Echokardiographie-Refresherkurs

München, 3.-4.7.2021, CME vorauss. 13

Kursleitung: $\quad$ PD Dr. med. Werner Zwehl, München

Kursort: $\quad$ Die Kochgarage

Nymphenburger Str. 25, 80335 München

Teilnahmegebühr: $\quad 480 €$ Nicht-Mitglied, $264 €$ BDI-Mitglied
Intensivkurs:

Kardiologie - Update 2021

Nürnberg, 9.-10.7.2021, CME vorauss. 16

Kursleitung: $\quad$ Prof. Dr. med. Matthias Pauschinger,

Kursort: $\quad$ Marmorsaal des Presseclub Nürnberg im Anwesen NÜRNBERGER Akademie Gewerbemuseumsplatz 2, 90403 Nürnberg

Teilnahmegebühr: $\quad$ Präsenz: $660 €$ Nicht-Mitglied, $363 €$ BDI Mitglied Livestream: $560 €$ Nicht-Mitglied, $308 €$ BDI-Mitglied

Intensivkurs:

Intensivkurs Innere Medizin - Refresher zur Facharztprüfung

Leipzig, 19.-23.7.2021, CME vorauss. 56

Kursleitung: $\quad$ Prof. Dr. med. Florian Lordick, Leipzig

Prof. Dr. med. Ulrich Laufs, Leipzig

Kursort: $\quad$ Großer Hörsaal im operativen Zentrum

Universitätsklinikum Leipzig

Liebigstr. 20, 04103 Leipzig

Teilnahmegebühr: $\quad 880 €$ Nicht-Mitglied, $484 €$ BDI Mitglied

Nachwuchs:

Internistische Summer School - Klinik Survival

Frankfurt, 27.-28.8.2021

Kursleitung: $\quad$ Dr. med. Cornelius Weiß, MPH, Wiesbaden

Kursort: Landesärztekammer Hessen

Hanauer Landstr. 152, 60314 Frankfurt/Main

Teilnahmegebühr: Präsenz: $120 €$ Nicht-Mitglied, $66 €$ BDI-Mitglied Livestream $60 €$ Nicht-Mitglied $33 €$ BDI-Mitglied

Praktischer Kurs:

Echokardiographie Grundkurs *gem. KBV-Richtl.

Pörtschach am Wörthersee, Österreich, 30.8.-3.9.2021, vorauss. 36 DFP Punkte

Kursleitung: $\quad$ Dr. med. Wolfgang Kirste, Schwabach

Dr. med. univ. Mathias Ausserwinkler, Salzburg Dr. med. univ. Mathias Ausserwinkler, Salz
Congress Center Wörthersee, Koschatsaal Hauptstr. 203 A-9210 Pörtschach a. Wörthersee

Teilnahmegebühr: $\quad 880 €$ Nicht-Mitglied, $484 €$ BDI Mitglied

\section{BDI Landesverbände 2021}

Internistentag:

Niedersachsen

Hannover, 5.6.2021

Leitung:

Ort:

Dr. med. Thomas Schmidt, Göttingen

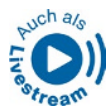

Stadionbrücke 4,30459 Hannover

Teilnahme:

kostenfrei

Fortbildung: Berlin/Brandenburg

"Neurologie für Internisten"

Online, 9.6.2021

Leitung:

Dr. med. Johannes Bruns, Berlin

Dr. med. Michael Witte, Berlin

Dr. med. Ekkard Beck, Grünheide

Teilnahme:

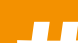




\section{Kurse \& Kongresse des BDI}

\author{
Intensivkurs: \\ Pneumologie - \\ Update 2021 \\ Bonn, 11.-12.6.2021
}
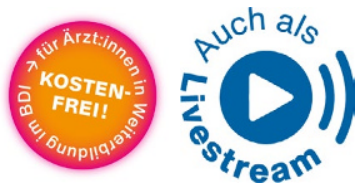

Kursleitung: PD Dr. med. Selcuk Tasci, Siegburg

Kursort: Universitätsclub Bonn e.V.

$\begin{array}{lll}\text { Zeit: } & \text { Freitag, 11.6.2021 } & 9: 30-17: 30 \\ & \quad \text { Samstag, 12.6.2021 } & 9: 00-16: 15\end{array}$

CME: $\quad$ Dieser Kurs wird mit vorauss. 16 Fortbildungspunkten

(Kategorie A) von der Landesärztekammer Nordrhein zertifiziert.

Teilnahme- Präsenz

gebühr: $\quad 660 €$ Nicht-Mitglied $363 €$ BDI Mitglied

Livestream

$560 €$ Nicht-Mitglied

$308 €$ BDI Mitglied

\section{Freitag, 11.6.2021}

9:30-10:15

Lungenembolie und Pulmonale

Hypertonie - Update 2021

Prof. Dr. med. Dirk Skowasch, Bonn

10:15-11:00

Interstitielle Lungenerkrankun-

gen - Wrap up

Prof. Dr. med. Dirk Skowasch, Bonn

11:00-11:15

Kaffeepause

11:15-12:00

Radiologische Differenzialdi-

agnose von Lungeninfiltraten

- Fallbasierte Systematik

PD Dr. med. Daniel Kütting, Bonn

12:00-12:45

Update Respiratorische

Infektionen 2021 - Alles außer

COVID-19

PD Dr. med. Selcuk Tasci, Siegburg

12:45-14:00

Mittagspause

14:00-14:45

Der unklare Lungenbefund - die

Tools der Bronchoskopie

PD Dr. med. Ute Oltmanns,

Pforzheim

14:45-15:30

Workup pleuraler Ergüsse

PD Dr. med. Ute Oltmanns,

Pforzheim

15:30-16:00

Kaffeepause

16:00-16:45

Schlafbezogene Atemstörungen

- Neues zu Diagnostik und

Therapie

PD Dr. med. Nikolaus Büchner,

Duisburg

16:45-17:30

Sauerstofftherapie, Highflow und

NIV - Nicht nur bei COVID-19?

PD Dr. med. Nikolaus Büchner,

Duisburg

9:00-9:15

Grußwort der Deutschen

Gesellschaft für Pneumologie

und Beatmungsmedizin

Prof. Dr. med. F. Joachim Meyer,

München

9:15-9:45

Pneumoquiz - Hätten Sie's

gewusst?

Prof. Dr. med. F. Joachim Meyer,

München

9:45-10:30

Personalisierte Therapie der COPD - Sind wir wirklich so weit? Prof. Dr. med. F. Joachim Meyer,

München

10:30-11:00

Kaffeepause

11:00-11:45

Symptom Atemnot - Eine interdisziplinäre Herausforderung

Prof Dr.med. Klaus Kenn,

Berchtesgaden

11:45-12:30

Rehabilitation bei Lungenerkran-

kungen - Wann, wie, wo?

Prof. Dr. med. Klaus Kenn,

Berchtesgaden

12:30-13:30

Mittagspause

13:30-14:15

Personalisierte Lungenkrebsbe-

handlung - Update 2021

Prof. Dr. med. Reinhard Büttner, Köln

14:15-15:00

Cor pulmonale - Verlegenheits-

diagnose oder Wirklichkeit?

Prof. Dr. med. Santiago Ewig

Bochum

15:00-15:30

Kaffeepause

15:30-16:15

Lungenfunktion: „for beginners

and experts"
BDI Online:

\section{Volkskrankheit der Inneren Medizin}

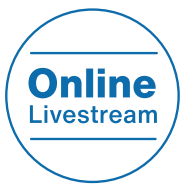

Online, 16.6.2021

Leitung: $\quad$ Prof. Dr. med. Ahmed Madisch, Hannover

Zeit: 17:00-19:35

Veranstalter: Berufsverband Deutscher Internisten e.V.

CME: Die Veranstaltung wird mit vorauss. 3 Fortbildungspunkten von der

Teilnahme: kostenfrei

Anmeldung: Veranstaltungsservice diaplan $\mathrm{GmbH}$ Alte Ziegelei 2-4, 51491 Overath Tel.: 02204 9731-04, Fax: 02204 9731-111 www.diaplan.de

Intensivkurs:

\section{Allgemeine Innere Medizin - Was ist \\ neu 2021?}
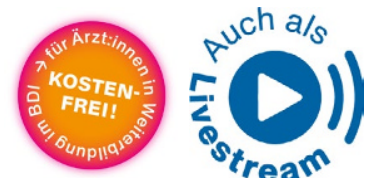

Dr. med. Nobert Mülleneisen, Köln

16:15-17:00

Asthma bronchiale - my way ...

Dr. med. Nobert Mülleneisen, Köln
Berlin, 18.-20.6.2021

Kursleitung: Prof. Dr. med. Bernhard Wörmann, Berlin

Kursort: $\quad$ Karl Storz SE \& Co. KG

$\begin{array}{llr}\text { Zeit: } & \text { Freitag, 18.6.2021 } & 16: 00-19: 30 \\ & \text { Samstag, 19.6.2021 } & 9: 00-17: 30 \\ & \text { Sonntag, 20.6.2021 } & 9: 00-12: 30 \\ \text { CME: } & \text { Dieser Kurs wird mit vorauss. 16 Fortbildungspunkten }\end{array}$

Besucher- und Schulungszentrum Berlin

Scharnhorststr. 3, 10115 Berlin (Mitte)

CME: $\quad$ Dieser Kurs wird mit vorauss. 16 Fortbildungspunkten

Teilnahme- Präsenz A) von der Ärztekammer Bivestream

gebühr: $\quad 660 €$ Nicht-Mitglied $\quad 560 €$ Nicht-Mitglied $363 €$ BDI Mitglied $\quad 308 €$ BDI Mitglied

\section{Freitag, 18.6.2021}

16:00-17:30

Kardiologie

- Herzinsuffizienz: Bewährtes und

Neues in Diagnostik und Therapie

17:30-18:00

Kaffeepause

18:00-19:30

Infektiologie/Gesundheitspolitik

- Covid 19-Wo stehen wir? Was haben

wir gelernt?

- Antibiotikaresistenz

- Herausforderungen an die Innere

Medizin

Prof. Dr. med. Andrew Ullmann, MdB,

Würzburg

9:00-10:30

Gastroenterologie

- Akute und chronische Pankreatitis

- Exokrine Pankreasinsuffizienz

- Zystische Pankreasläsionen

- Cholelithiasis

Prof. Dr. med. Jonas Rosendahl, Halle

\section{0:30-11:00}

Kaffeepause

11:00-12:30

Geriatrie

N. N.

12:30-14:00

Mittagspause
Prof. Dr. med. Frank Edelmann, Berlin

\section{Samstag, 19.6.2021}

14:00-15:30

Diabetologie

- Neue Leitlinien und ihre digitale

Implementierung für die Therapie des

Typ-2-Diabetes"

Prof. Dr. med. Dirk Müller-Wieland,

Aachen

15.30-16:00

Kaffeepause

16:00-17:30

Onkologie

- Multiples Myelom

- Lungenkarzinom

- Prostatakarzinom

Prof. Dr. med. Bernhard Wörmann, Berlin

\section{Sonntag, 20.6.2021}

9:00-10:30

Nephrologie

- Diabetische Nephropathie

- Glomerulonephritis

- Akute Nierenschädigung

- Dialyse

Prof. Dr. med. Matthias Girndt, Halle

10:30-11:00

Kaffeepause

11:00-12:30

Pneumologie

- Asthma bronchiale

- Schlafapnoe

- Langzeitbeatmung/Weaning

Prof. Dr. med. Christian Grohé, Berlin

Änderungen vorbehalten 


\section{Intensivkurs: \\ Nephrologie/ Diabetologie - Update 2021}

\author{
Weimar, 25,-26.6.2021
}

Kursleitung: Prof. Dr. med. Reinhard Fünfstück, Weimar

PD Dr. med. Kornelia Konz, Wiesbaden

$\begin{array}{ll}\text { Kursort: } & \text { Dorint Am Goethepark Weimar } \\ & \text { Beethovenplatz 1/2,99423 Weim }\end{array}$

Zeit:

Freitag, 25.6.2021

9:30-17:30

Samstag, 26.6.2021

9:00-17:10

CME: $\quad$ Dieser Kurs wird mit vorauss. 16 Fortbildungspunkten (Kategorie A) von der Landesärztekammer Thüringen zertifziert.

Teilnahme- Präsenz

gebühr: $\quad 660 €$ Nicht-Mitglied $363 €$ BDI Mitglied

Livestream

$560 €$ Nicht-Mitglied

$308 €$ BDI Mitglied

\section{Freitag, 25.6.2021}

Moderation: Prof. Dr. med. Reinhard Fünfstück

9:30-9:50

Begrüßung und Einführung

Prof. Dr. med. Reinhard Fünfstück;

Weimar

Ralf Kirsten, Bürgermeister der Stadt

Weimar

9:50-10:30

Mikrobiologisch-infektiologi-

sche Qualitätsstandards bei

Harnwegsinfektionen

Prof. Dr. med. Reinhard Fünfstück,

Weimar

10:30-11:15

Behandlungsstrategien bei

Patienten mit CKD

Prof. Dr. med Mark Dominik Alscher,

Stuttgart

11:15-11:45

Kaffeepause

11:45-12:15

Risikofaktor: Hypertonie

Prof. Dr. med. Reinhard Fünfstück,

Weimar

12:15-13:00

Polypharmazie bei CKD -

vermeidbar?

Prof. Dr. med. Sylvia Stracke,

Greifswald

13:00-14:00

Mittagspause

14:00-14:45

Welches Dialyseverfahren für

welchen Patienten?

Prof. Dr. med Mark Dominik Alscher, Stuttgart

14:45-15:30

Dialyse im fortgeschrittenen

Lebensalter - was ist anders?

Prof. Dr. med. Clemens Grupp,

Bamberg

15:30-16:00

Kaffeepause

16:00-16:45

Kognitive Veränderungen

unter den Bedingungen einer

Niereninsuffizienz

Prof. Dr. med. Sylvia Stracke,

Greifswald
16:45-17:30

Dialyse Abbruch: Ethische und rechtliche Probleme

Prof. Dr. med. Clemens Grupp

Bamberg

\section{Samstag, 26.6.2021}

Moderation: PD Dr. med. Kornelia Konz

9:00-10:00

Therapie des Typ-2-Diabetes, die neuen Leitlinien (ADA, EASD, ESC)

PD Dr. med. Kornelia Konz

Wiesbaden

10:00-11:00

Prof. Dr. med. Martin Pfohl, Duisburg

11:00-11:30

Kaffeepause

11:30-12:30

Diabetestherapie beim älteren

Menschen

Dr. med. Norbert Schütz, Wiesbaden

12:30-13:15

Hautveränderungen bei Diabetes

Dr. med. Galina Balakirski, Bonn

13:15-14:15

Mittagspause

14:15-15:00

Diabetologie in Klinik und Praxis.

Knotenpunkt oder Sackgasse?

Prof. Dr. med. Martin Pfohl, Duisburg

15:00-15:45

Diabetesassoziierte gastrointesti-

nale Erkrankungen

Prof. Dr. med. Felix Gundling,

Bamberg

15:45-16:15

Kaffeepause

16:15-17:00

Sars-CoV2 und Diabetes

PD Dr. med. Kornelia Konz

Wiesbaden

17:00-17:10

Schlussworte und Verabschiedung
Insulintherapie: Update 2021

\section{Intensivkurs: \\ Kardiologie - Update 2021}

\section{Nürnberg, 9.-10.7.2021}

Kursleitung: Prof. Dr. med. Matthias Pauschinger, Nürnberg

Kursort: $\quad$ Marmorsaal des Pressclub Nürnberg im Anwesen NÜRNBERGER Akademie

$\begin{array}{lll} & \text { Gewerbemuseumsplatz 2, 90403 Nürnberg } \\ \text { Zeit: } & \text { Freitag, 9.7.2021 } & 9: 30-17: 30 \\ & \text { Samstag, 10.7.2021 } & 9: 00-17: 00 \\ \text { CME: } & \text { Dieser Kurs wird mit vorauss. 16 Fortbildungspunk } & \text { (Kategorie A) von der Bayerischen Landesärztek } \\ & \text { zertifiziert. } & \\ \text { Teilnahme- } & \text { Präsenz } & \text { Livestream } \\ \text { gebühr: } & 660 € \text { Nicht-Mitglied } & 560 € \text { Nicht-Mitglied } \\ & 363 € \text { BDI Mitglied } & 308 € \text { BDI Mitglied }\end{array}$

Freitag, 9.7.2021

9:30-10:15

$\mathrm{PCl}$ beim geriatrischen Patienten, was muss man beachten?

Prof. Dr. med. Harald Rittger, Fürth

10:15-11:00

Chronischer Gefäßverschluss

in der Koronarangiographie

(CTO) - was ist indiziert und was

ist machbar?

Fadil Ademaj, Nürnberg

11:00-11:30

Kaffeepause

11:30-12:15

Akutes Koronarsyndrom, welche

Rolle spielt das Koronar-CT

PD Dr. med. Karsten Pohle, Nürnberg

12:15-13:00

Versorgung von Patienten mit akutem Koronarsyndrom in der

Pandemie - Was ist passiert?

Dr. med. Dennis Eckner, Nürnberg

13:00-14:00

Mittagspause

14:00-14:45

MINOCA - welche medika-

mentöse Behandlungsstrate-

gien ergeben sich bei diesem

Krankheitsbild?

Dr. med. univ. Kristinko Martinovic Nürnberg

14:45-15:30

Update Aortenklappenstenose

Prof. Dr. med. Matthias Pauschinger,

Nürnberg

15:30-16:00

Kaffeepause

16:00-16:45

PFO Verschluss - welcher Patient profitiert von diesem Eingriff?

Riza Sahin, Nürnberg

16:45-17:30

Interventionelle Therapie der Mitralklappeninsuffizienz - Hilft die Vergrößerung des Baukastens?

Prof. Dr. med. Marcus Hennersdorf, Heilbronn

\section{Samstag, 10.7.2021}

9:00-9:45

Vorhofflimmern und PCI mit

Stentimplantation - Was, wie und wie lange?

Dr. med univ. Andrea Brinker-Paschke, Nürnberg

9:45-10:30

Nicht anhaltende VTs im Langzeit EKG - Was ist zu tun?

Prof. Dr. med. Christian von Bary, München

10:30-11:00

Kaffeepause

11:00-11:45

Herzinsuffizienz und Vorhofflimmern - gibt es neue Aspekte in der Therapie?

Prof. Dr. med Thomas Deneke, Bad Neustadt

11:45-12:30

Update Guidelines Heart Failure Prof. Dr. med. Stephan Achenbach Erlangen

12:30-13:00

Mittagspause

13:30-14:15

Diastolische Herzinsuffizienz,

Licht am Horizont?

Prof. Dr. med. Wolfgang von Scheidt, Augsburg

14:15-15:00

Telemedizin in der Kardiologie hilft der neue GBA Beschluss?

Prof. Dr. med. Dieter Ropers,

Nürnberg

15:00-15:30

Kaffeepause

15:30-16:15

Extrakorporaler life support -

Wann für welchen Patienten?

Dr. med. Matthias Fittkau, Nürnberg

16:15-17:00

Therapie des kardiogenen

Schocks. Bedeutung des ECLS?

Prof. Dr. med. Robert Schwinger, Weiden 


\section{Praktischer Kurs: \\ Farbdoppler-Echokardiographie- Refresherkurs}

München, 3.-4.7.2021

Wissenschaftliche

Kursleitung: PD Dr. med. Werner Zwehl, München

Kursort: Die Kochgarage

Nymphenburger Str. 25; 80335 München

Zeit: $\quad$ Samstag, 3.7.2021 9:00-17:30

$\begin{array}{ll} & \text { Sonntag, 4.7.2021 } \\ \text { CME: } & \text { Dieser Kurs wird mit vorauss. } 13 \text { Fortbildungspunkten }\end{array}$

(Kategorie A) von der Bayerischen Landesärztekammer zertifiziert

Teilnahme- $\quad 480 €$ Nicht-Mitglied

gebühr: $\quad 264 €$ BDI Mitglied

\section{BDI Online:}

\section{Therapierefraktäre Behandlungssituationen häufiger internistischer Krankheitsbilder}

\author{
Online, 14.7.2021 \\ Leitung: $\quad$ Prof. Dr. med. Ahmed Madisch, Hannover \\ Zeit: $\quad$ 17:00-19:35 \\ Veranstalter: Berufsverband Deutscher Internisten e.V. \\ CME: Die Veranstaltung wird mit vorauss. 3 Fortbildungspunk- \\ ten von der Ärztekammer Niedersachsen zertifiziert. \\ Teilnahme: kostenfrei \\ Anmeldung: Veranstaltungsservice diaplan $\mathrm{GmbH}$ \\ Alte Ziegelei 2-4, 51491 Overath \\ Tel.: 02204 9731-04, Fax: 02204 9731-111 \\ www.diaplan.de
}

\section{Refresherkurs:}

\section{Intensivkurs Innere Medizin - Refresher zur Facharztprüfung}

\section{Leipzig, 19.-23.7.2021}

Kursleitung: Prof. Dr. med. Florian Lordick, Leipzig

Prof. Dr. med. Ulrich Laufs, Leipzig

Kursort: $\quad$ Großer Hörsaal im operativen Zentrum

Universitätsklinikum Leipzig

Liebigstr. 20, 04103 Leipzig

Zeit: $\quad$ Montag-Freitag, 19.-23.7.2021 8:15-18:00

CME Dieser Kurs wird mit vorauss. 56 Fortbildungspunkten (Kategorie A) von der Landesärztekammer Sachsen zertifiziert.

Teilnahme- $\quad 880 €$ Nicht- Mitglied

gebühr: $\quad 484 €$ BDI Mitglied

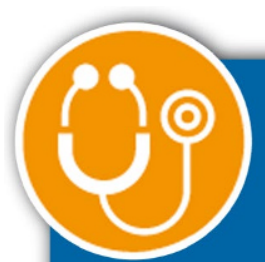

Kongresse und Fortbildungen
Online: www.bdi.de
eMail: fortbildung@bdi.de
Tel:: 0611 18133-21/22
Fax: 0611 18133-23

Buchen Sie doch einfach direkt!

\section{Klinik Survival}

Frankfurt, 27.-28.8.2021

Kursleitung: Dr. Cornelius Weiß, MPH, Wiesbaden Kursort: Landesärztekammer Hessen

Hanauer Landstraße 152

Zeit: $\quad$ Freitag, 27.8.2021

Samstag, 28.8.2021

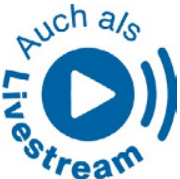

10:00-17:45

9:00-17:15

Veranstalter: Berufsverband Deutscher Internisten e.V. Bündnis Junge Internisten

$\begin{array}{lll}\text { Teilnahme- } & \text { Präsenz } & 120 € \text { Nicht-Mitglied } \\ \text { gebühr: } & & 66 € \text { BDI Mitglied } \\ & \text { Livestream } & 60 € \text { Nicht-Mitglied } \\ & & 33 € \text { BDI Mitglied }\end{array}$

Freitag, 27.8.2021

10:00-10:30

Ankunft und Registrierung der Teilnehmer

10:30-11:00

Begrüßung

11:00-11:45

Rheumatologie

Was ist Rheuma, was nicht?

Und wenn ja, was muss ich tun?

11:45-12:30

\section{Berufspolitik Teil I}

Wer vertritt mich eigentlich wo, und wieso ist das wichtig?

12:30-13:15

\section{Kardiologie Teil I}

Herzinsuffizienz, sicher im Akutfall und im chronischen Verlauf handeln

13:15-14:15

Mittagspause

14:15-15:00

Kardiologie Teil II

Am Anfang war das EKG, vom EKG

zur Diagnose und Therapie

15:00-15:45

Endokrinologie

Essentials, den endokrinologischen

Fall erkennen lernen

15:45-16:15

Kaffeepause

16:15-17:00

Diabetologie

„Das mach ich alles selbstständig

Herr Doktor, nie Probleme" -

Therapieeinleitung und Anpassung

der diabetischen Therapie an den

einzelnen Patienten

\section{7:00-17:45}

Angiologie

Dos and Don'ts - von Antikoagu-

lation bis Revaskularisation

\section{Samstag, 28.8.2021}

9:00-9:45

Gastroenterologie Teil I

Unklares Abdomen, Galle-Leber oder Pankreas? -

Labor und Klinik richtig deuten

9:45-10:30

Gastroenterologie Teil II

Leberzirrhose, praktische Tipps

zum Management der

Diagnostik und Therapie

10:30-11:00

Kaffeepause

11:00-11:45

Pneumologie

Pneumo-Basics für die Innere -

das pneumologische Survival-Kit für den Klinikstart

11:45-12:30

Nephrologie

Kreatinin hoch, was nun?

12:30-13:00

Berufspolitik II

„Kein Kampf gegen Windmühlen warum sich Berufspolitik

auch für junge Ärzt*innen lohnt ${ }^{*}$

13:00-14:00

Mittagspause

14:00-15:00

Fit für den Dienst Teil I

Bei Anruf Notfall - Interaktive prakt.

Fallbeispiele

- Die Herzfrequenz ist irgendwie

zu hoch

- Der Druck geht nicht runter!

- Die kriegt keine Luft!

15:00-15:30

Kaffeepause

15:30-16:50

Fit für den Dienst Teil II

Bei Anruf Notfall - Interaktive prakt.

Fallbeispiele

- Der bricht blutig!

- Die ist irgendwie weggetreten

- Der Zucker ist so hoch/niedrig

- Der hat so Schmerzen, was soll ich geben?

16:50-17:15

Evaluation

Änderungen vorbehalten 


\section{Seminarwoche:}

\section{Seminarwoche Innere Medizin kompakt}

\section{Pörtschach am Wörthersee, Österreich,} 29.8.-3.9.2021

Leitung: $\quad$ Prof. Dr. med. Andreas Tromm, Hattingen

$\begin{array}{ll} & \text { Prof. Dr. med. Bernhard Wörmann, Berlin } \\ \text { Kursort: } & \text { Congress Center Wörthersee }\end{array}$

Kuuptstr. 203,9210 Pörtschach a. Wörthersee/Österreich

Zeit: $\quad$ Sonntag, 29.8.2021 Anreise \& Eröffnungsvortrag

Montag - Freitag, 30.8.-3.9.2021 9:00-12:30 \&

15:00-18:30

CME: $\quad$ Diese Seminarwoche wird mit vorauss. 40-DFP Punkten von der Österreichischen Ärztekammer approbiert sowie mit vorauss. 30 Credits von der Schweizerischen Gesellschaft für Allgemeine Innere Medizin anerkannt! DMP Anerkennung wird beantragt

Teilnahmegebühr:

$\begin{array}{lll} & \text { Nicht-Mitglied } & \text { BDI Mitglied } \\ \text { Seminarwoche } & 620 € & 341 € \\ \text { Tageskarte } & 220 € & 121 € \\ \text { Assistenzpersonal } & 250 € & 250 €\end{array}$

\section{Praktischer Kurs:}

\section{Echokardiographie Grundkurs *gem. KBV-Richtl.}

\begin{tabular}{|c|c|}
\hline Kursleitung: & $\begin{array}{l}\text { Dr. med. Wolfgang Kirste, Schwabach } \\
\text { Dr. med. univ. Mathias Ausserwinkler, Salzburg }\end{array}$ \\
\hline Kursort: & $\begin{array}{l}\text { Congress Center Wörthersee } \\
\text { Hauptstr. 203, A-9210 Pörtschach a. Wörthersee, } \\
\text { Österreich }\end{array}$ \\
\hline Zeit: & $\begin{array}{ll}\text { Montag-Donnerstag, 30.8.-2.9.2021 } & 9: 00-18: 30 \\
\text { Freitag, 3.9.2021 } & 9: 00-12: 30\end{array}$ \\
\hline & $\begin{array}{l}\text { Dieser Kurs wird mit vorauss. 36-DFP Punkten von der } \\
\text { Österreichischen Ärztekammer approbiert. }\end{array}$ \\
\hline $\begin{array}{l}\text { Teilnahme- } \\
\text { gebühr: }\end{array}$ & $\begin{array}{l}880 € \text { Nicht-Mitglied } \\
484 € \text { BDI Mitglied }\end{array}$ \\
\hline
\end{tabular}

\section{Veranstaltungen der BDI-Landesverbände}

\section{Internistentag \\ Niedersachsen}

Hannover, 5.6.2021

\begin{tabular}{|c|c|}
\hline $\begin{array}{l}\text { Leitung } \\
\text { Ort: }\end{array}$ & $\begin{array}{l}\text { Dr. med. Thomas Schmidt, Göttingen } \\
\text { Klinikum KRH Siloah } \\
\text { Stadionbrücke 4, } 30459 \text { Hannover }\end{array}$ \\
\hline Zeit: & 9:00-16:00 \\
\hline CME: & $\begin{array}{l}\text { Diese Veranstaltung wird zur Zertifizierung bei der } \\
\text { Landesärztekammer eingereicht. }\end{array}$ \\
\hline Teilnahme: & kostenfrei \\
\hline Anmeldung: & $\begin{array}{l}\text { Veranstaltungsservice diaplan GmbH } \\
\text { Alte Ziegelei 2-4, 51491 Overath } \\
\text { Tel.: } 02204 \text { 9731-04, Fax: 02204 9731-111 } \\
\text { www.diaplan.de }\end{array}$ \\
\hline
\end{tabular}

Fortbildung

Berlin/Brandenburg

„Neurologie für Internisten"

Online, 9.6.2021

Leitung: Dr. med. Johannes Bruns, Berlin

Dr. med. Michael Witte, Berlin

Ort: $\quad$ Online

Zeit: $\quad$ 18:00-20:30

Teilnahme: kostenfrei

Anmeldung: Veranstaltungsservice diaplan $\mathrm{GmbH}$ Alte Ziegelei 2-4, 51491 Overath Tel.: 02204 9731-04

Fax: 02204 9731-111 www.diaplan.de

\section{Internistentag \\ Westfalen-Lippe \\ Dortmund, 26.6.2021 \\ Leitung: $\quad$ Dr. med. Karl-Heinz Pfetsch, Salzkotten \\ Ort: $\quad$ Radisson Blu Hotel Dortmund \\ An der Buschmühle 1, 44139 Dortmund \\ Zeit: $\quad$ 9:00-16:00 \\ CME: Diese Veranstaltung wird zur Zertifizierung bei der \\ Teilnahme: kostenfrei \\ Anmeldung: Veranstaltungsservice diaplan $\mathrm{GmbH}$ \\ Alte Ziegelei 2-4, 51491 Overath \\ Tel.: 02204 9731-04, Fax: 02204 9731-111 \\ www.diaplan.de}

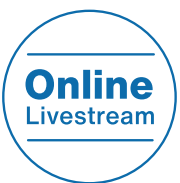

\section{Internistentag}

\section{Baden-Württemberg}

\section{Stuttgart, 24.7.2021}

Leitung: Dr. med. Norbert Smetak, Kirchheim

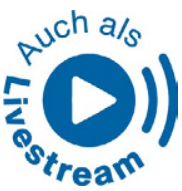

Ort: $\quad$ IHK - Industrie- und Handelskammer

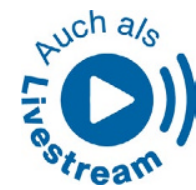

Ort: $\quad$ IHK - Indust

Jägerstr. 30, 70174 Stuttgart

Zeit: $\quad$ 9:00-15:30

CME: Die Veranstaltung wurde bei der Landesärztekammer

Baden-Württemberg zur Zertifizierung eingereicht

Teilnahme: kostenfrei

Anmeldung: Veranstaltungsservice diaplan $\mathrm{GmbH}$

Alte Ziegelei 2-4, 51491 Overath

Tel.: 02204 9731-04, Fax: 02204 9731-111

www.diaplan.de

\section{Internistentag:}

Schleswig-Holstein

Kiel, 7.8.2021

Leitung: $\quad$ Dr. med. Klaus Heger, Glücksstadt

Ort: $\quad$ Steigenberger Conti Hansa Schloßgarten 7,24103 Kiel

Zeit: $\quad 9: 00-16: 00$

CME: Die Veranstaltung wird zur Zertifizierung bei der

Teilnahme: Landesärztek

Anmeldung: Veranstaltungsservice diaplan $\mathrm{GmbH}$ Alte Ziegelei 2-4, 51491 Overath Tel.: 02204 9731-04, Fax: 02204 9731-111 www.diaplan.de 


\section{BDI Online}
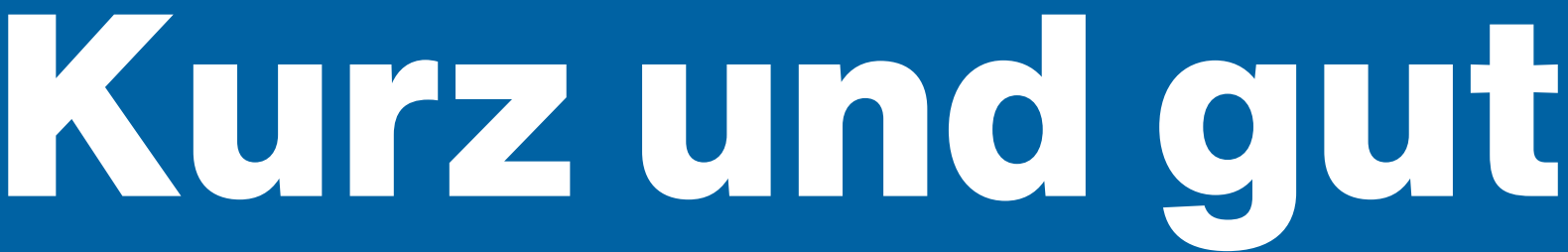

Unser neues Online-Kursformat bietet kostenfreie Weitertbildung in kompakten 21/2 Stunden - für überall.

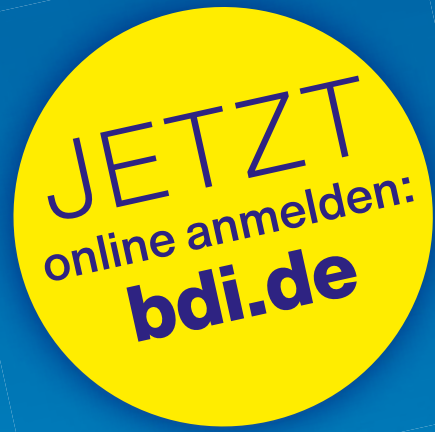

$\rightarrow$ Volkskrankheiten der Inneren Mredizin $\mathrm{Mi}, 16.6 .2021$ vorauss. 3 CME-Punkte - kostenfrei

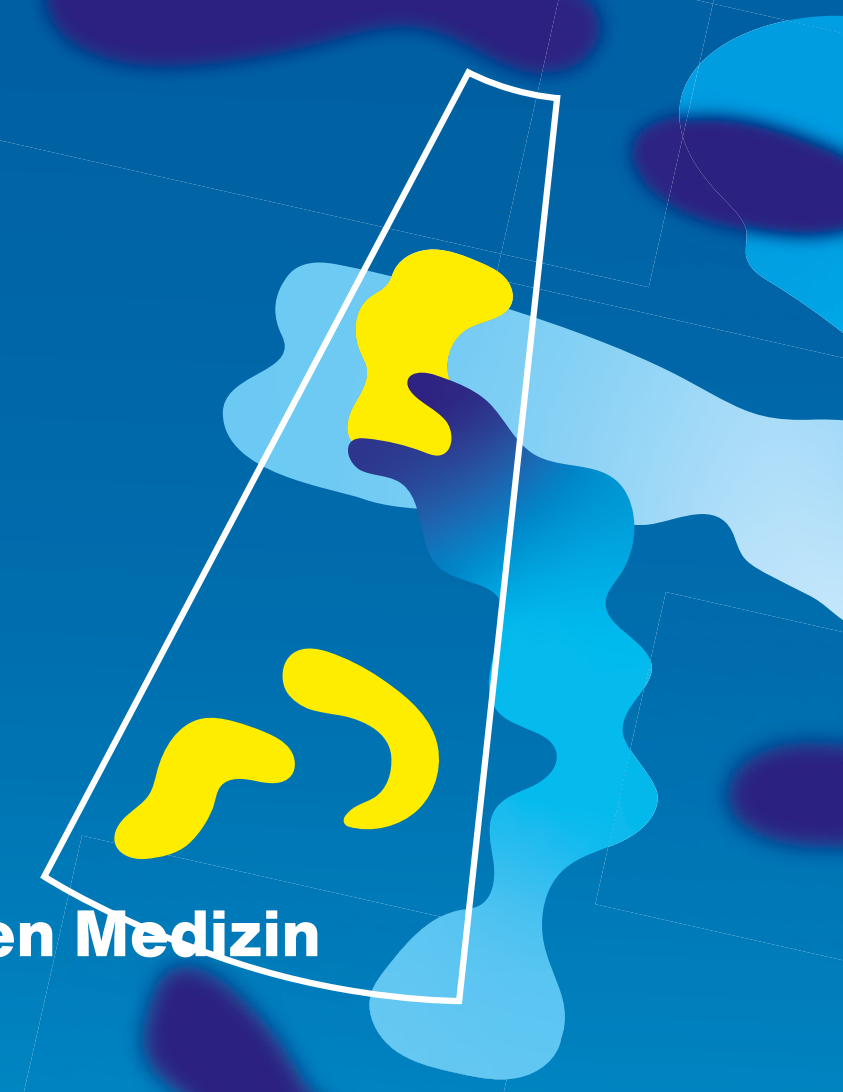

$\rightarrow$ Therapierefraktäre Behandlungssituation häufiger internistischer Krankheitsbilder $\mathrm{Mi}, 14.7 .2021$ vorauss. 3 CME-Punkte - kostenfrei

\section{$\rightarrow$ Update Leitlinien}

$\mathrm{Mi}, 1.9 .2021$

vorauss. 3 CME-Punkte - kostenfrei 